\title{
Challenges Faced by Radiography Students During Clinical Training
}

\author{
Kyei K. A., Antwi W. K., Bamfo-Quaicoe K., Offei R. O. \\ Department of Radiography, School of Biomedical and Allied Health Sciences, University of Ghana, Korle-Bu, Accra, Ghana
}

Email address:

adesco41@hotmail.com (K. A. Kyei), kakyei@chs.ug.edu.gh (K. A. Kyei)

\section{To cite this article:}

Kyei K. A., Antwi W. K., Bamfo-Quaicoe K., Offei R. O.. Challenges Faced by Radiography Students During Clinical Training. Clinical Medicine Research. Special Issue: Radiographic Practice Situation in a developing Country. Vol. 4, No. 3-1, 2015, pp. 36-41. doi: 10.11648/j.cmr.s.2015040301.18

\begin{abstract}
Background: Clinical training forms part of the requirements of every radiography student, for the award of Bachelor's degree at the University of Ghana. The effectiveness of clinical training is responsible for the competency level that would be demonstrated by qualified radiography students. However, the capabilities of department to provide adaptive and well managed clinical training for undergraduate students have been reported as a limiting factor. Aim: The purpose of the study was to identify challenges facing the student radiographers during clinical training. Methods: The study was a quantitative one which employed a descriptive survey approach. The survey comprised of levels 300 and 400 students of the department of radiography which gathered forty-two (42) participants. A questionnaire was used to collect data for the study. Data obtained was summarized as frequencies, percentages, means and standard deviations using SPSS version 16.0. Results: The study revealed challenges faced by radiography students such as the gap between theory and practices, inadequate exposure to certain specialized procedures and time allotted to each treatment room. Conclusion: The study showed that clinical training can be enhanced by providing enough equipments and clinical areas for students, also films and cassettes must be made available before the date and time of clinical training. Finally, the theory aspects of clinical training must be in tune with the practice to enhance effective learning experience by students.
\end{abstract}

Keyword: Clinical Training, Challenges, Skill Development; Supportive Learning

\section{Background}

Undertaking clinical training is part of the requirements expected of every radiography student, for the award of Bachelor's degree at the University of Ghana. Therefore, clinical training becomes a compulsory component of the level 300 and 400 training syllabus. Several challenges have been documented in some countries regarding these clinical trainings [1, 2]. The report of Simpson [1] of the Newcastle Mater Misericordiae Hospital (NMMH) Department of Radiation Oncology, spoke about Medical Radiation Science (MRS) student training. At a time, the majority of the staff felt that the issue of student training needed attention, the department had failed to provide a suitable educational programme for undergraduate radiation therapists [1]. The students reported that "they did not feel welcomed when they first went to the department for their clinical placements" [1].

Baume [3] reviewed the status of the radiography profession on many fronts such as, staffing, training and equipment. The long waiting times for access to Radiation Therapy (RT) services were attributed to the shortage of Radiation Therapists (RTs), Radiation Oncologists and Medical Physicists. The shortage of RTs was investigated in depth, highlighting staff attrition rates, student intake and student attrition. Student attrition was attributed to poor experiences in a clinical facility. The capabilities of departments to provide adaptive and well managed clinical placements for undergraduate students were seen as a limiting factor, [3]. Particularly, due to lack of staff to mentor the students and their increasing numbers. Many papers and text, over the years, have rather addressed the issues of clinical enhancement and preceptorship of various undergraduate students from various health disciplines, primarily nursing $[1,2]$.

Likewise, the radiography program being run at the University of Ghana may probably be facing similar challenges. Unfortunately, however, there exists no literature regarding students' clinical experiences during the 
radiography program in Ghana.

Clinical training plays an important role in the training of students in the Health Sciences. The effectiveness of clinical training is responsible for the competency level that would be demonstrated by qualified radiography students in the care and management of patients with little or no supervision, [3]. However, it appears that student radiographers at the site face numerous challenges during clinical trainings which have not been documented. These challenges may go a long way to hamper the progress and quality of students in clinical training. These factors influenced the conduct of this research. The study attempted to reveal the existing challenges faced by radiography students during clinical training and ways to address them.

\section{Methodology}

The study was a quantitative one which will employ a descriptive survey approach. The study was conducted in the School of Biomedical and Allied Health Sciences, in one of the Teaching Hospitals in Ghana where the radiography students are trained. The research included all level 300 and 400 student radiographers in the study site because they had experience and knowledge on clinical rotation. The first and second year students were excluded because they had little or no experience on the clinical settings. In all there were 42 radiography students from the two levels (Level 300 and level 400) and this formed the sample of the study.

A self-structured questionnaire was used to collect data from respondents consisting of four sections, A-D, that comprised of demographic data all through to questions on theory and practice gap existing during clinical trainings. The questionnaires were administered to all students who were within the inclusion criteria and consented to participate. The collected data was analyzed using Statistical Package for Social Scientists (SPSS version 16.0). The data was coded using numeric values (e.g. marital status, Age group, gender), for socio-demographic categorical data (i.e. age group, sex), summary tables of counts and percentages were presented with respect to these characteristics. In some cases, graphical presentations were provided to highlight the level of differences. In cases of sparse data (less than 5\% observation), to avoid bias on the conduct of analysis, the assessments of respondents in such categories were excluded.

Approval was obtained from the Ethical Review and Protocol Committee of a higher institution. The ethics approval was supported by written permission for the study to be conducted at the study site with the ethical standard of confidentiality being upheld. All participants gave informed consent prior to the commencement of the study and each data collection activity. The study did not involve any risk or harm as it sought to seek information from participants of their work related stress.

\section{Results}

The study sampled $42(100 \%)$ respondents, out of which most $34(81 \%)$ were found in the age group 19-23 and the least $(7.1 \%)$ in age group 29-33. None of the respondents had age above 33 years. The study sampled demonstrated males dominance $25(59.5 \%)$ as compared to females. More than half of the respondents $23(54.8 \%)$ in the study were in level 300 while $19(45.2 \%)$ were in level 400 . Majority of the respondents $40(95.2 \%)$ were single while $2(4.8 \%)$ were married.

Table 1. Distributions of student's perception of radiographers at clinical settings

\begin{tabular}{|c|c|c|c|c|}
\hline Perceptions & Strongly Agree & Agree & Disagree & Strongly disagree \\
\hline The preparation for clinical training in the university was useful. & $16(38.1)$ & $24(57.1)$ & $1(2.4)$ & $1(2.4)$ \\
\hline Orientation by supervisors/clinical staff was adequate. & $1(2.4)$ & $23(54.8)$ & $16(38.1)$ & $2(4.8)$ \\
\hline Were you expected at your clinical room? & $12(28.6)$ & $22(52.4)$ & $7(16.7)$ & $1(2.4)$ \\
\hline The duty roaster was available in advance. & $20(47.6)$ & $17(40.5)$ & $4(9.5)$ & $1(2.4)$ \\
\hline $\begin{array}{l}\text { The practice experience and supervision offered were } \\
\text { appropriate to your expectation? }\end{array}$ & $4(9.5)$ & $22(52.4)$ & $14(33.3)$ & $2(4.8)$ \\
\hline The clinical staff was willing and available to assist learning? & $6(14.3)$ & $28(66.7)$ & $7(16.7)$ & $1(2.4)$ \\
\hline The staff took your errors and mistakes lightly. & $4(9.5)$ & $27(64.3)$ & $8(19.1)$ & $3(7.1)$ \\
\hline
\end{tabular}

A total of $95.2 \%$ of the respondents agree that the preparation for clinical training in the university was useful where as $54.8 \%$ of the respondents 'agree' that orientation by supervisors/clinical staff was adequate.

Table 2. Distributions for available resources for student radiographers

\begin{tabular}{lllll}
\hline Resources & Very adequate & Adequate & Inadequate & Not available \\
\hline X-ray Equipment & $5(11.9)$ & $15(35.7)$ & $22(52.4)$ & $0(0.0)$ \\
Cassettes and films & $10(23.8)$ & $22(52.4)$ & $10(23.8)$ & $0(0.0)$ \\
Anatomical marker & $1(2.4)$ & $13(31.0)$ & $18(42.9)$ & $10(23.8)$ \\
Monitoring devices & $0(0.0)$ & $6(14.3)$ & $11(26.2)$ & $25(59.6)$ \\
\hline
\end{tabular}

More than half of the respondents $22(52.4 \%)$ reported that X-ray equipments were 'inadequate' at the clinical settings while $35.7 \%$ reported that it was 'adequate'. Majority of the respondents $76.2 \%$ reported that cassettes 
Table 3. Distributions for imaging rooms respondents have been to

\begin{tabular}{llll}
\hline Imaging modalities & Yes & No & Total \\
\hline MRI & $27(64.3)$ & $15(35.7)$ & $42(100.0)$ \\
CT & $26(61.9)$ & $16(38.1)$ & $42(100.0)$ \\
FLOUROSCOPY & $29(69.0)$ & $13(31.0)$ & $42(100.0)$ \\
HSG & $34(81.0)$ & $8(19.0)$ & $42(100.0)$ \\
DENTAL & $13(31.0)$ & $29(69.0)$ & $42(100.0)$ \\
IVU & $25(59.5)$ & $17(40.5)$ & $42(100.0)$ \\
MAMMOGRAM & $14(33.3)$ & $28(66.7)$ & $42(100.0)$ \\
GERAD & $42(100.0)$ & $0(0.0)$ & $42(100.0)$ \\
WARD THEATRE & $7(16.7)$ & $35(83.3)$ & $42(100.0)$ \\
ULTRASOUND & $23(54.8)$ & $19(45.2)$ & $42(100.0)$ \\
\hline
\end{tabular}

Majority of the respondents (31\%) had not been allocated to the dental room and $(66.7 \%)$ the mammography imaging room. An overwhelming $83 \%$ of the respondents had never being on theatre radiology.

Table 4. Adequacy of time allocation to clinical rotation

\begin{tabular}{|c|c|c|c|c|c|}
\hline Imaging modalities & Very Adequate & Adequate & Inadequate & Very Inadequate & Total \\
\hline MRI & $1(2.3)$ & $11(25.6)$ & $13(30.2)$ & $7(16.3)$ & $42(100.0)$ \\
\hline FLOUROSCOPY & $1(2.3)$ & $11(25.6)$ & $16(37.2)$ & $8(18.6)$ & $42(100.0)$ \\
\hline HSG & $1(2.3)$ & $19(44.2)$ & $18(41.9)$ & $1(2.3)$ & $42(100.0)$ \\
\hline IVU & $2(4.7)$ & $14(32.6)$ & $13(30.2)$ & $6(14.0)$ & $42(100.0)$ \\
\hline MAMMOGRAPHY & $1(2.3)$ & $7(16.3)$ & $6(14.0)$ & $19(44.2)$ & $42(100.0)$ \\
\hline GERAD & $7(16.3)$ & $32(74.4)$ & $2(4.7)$ & $2(4.7)$ & $42(100.0)$ \\
\hline WARD THEATRE & $1(2.3)$ & $4(9.3)$ & $3(7.0)$ & $7(16.3)$ & $42(100.0)$ \\
\hline DENTAL & $3(7.0)$ & $7(16.3)$ & $7(16.3)$ & $8(18.6)$ & $42(100.0)$ \\
\hline ULTRASOUND & $0(0.0)$ & $4(9.3)$ & $13(30.2)$ & $15(34.9)$ & $42(100.0)$ \\
\hline
\end{tabular}

Apart from general radiography, most of the respondents indicated they had not been all the other imaging modalities.

\section{Establishing the Theory and Practice Gap}

Respondents were asked to report on the relationship between theory taught in class and practice and also provide some suggestions to improving the clinical experience of student Radiographers. There were mixed views on among respondents as the relationship between theory and practice. Some reported that there was no relationship while others reported that there is a relationship. Respondents suggested several ways of improving clinical experience such as using the same lecturers who teach the theory to handle the clinical areas and also theory should be immediately followed by practice. Conducting of continuous professional training was also suggested, improving facilities and time allocation.

\section{Discussion}

The study sampled $42(100 \%)$ respondents. Majority of the respondents in this study were within the ages of $19-23(81 \%)$, $11 \%$ within $24-28$ years and the least $7.1 \%$ in age group 29 33 years. None of the respondents had age above 33 years as shown in. Respondents who were above 33 years could be those who were admitted as matured students and had certificate or diploma in radiography before applying to the degree program. The study also sampled more males $59.5 \%$ compared to females $40.5 \%$. Majority of the respondents
$54.8 \%$ were level 300 and $45.2 \%$ form those at level 400 . It is indicative that level 300 are more, whiles level 400 of the eligible sample respondents represented are the minority in level 400 .

\subsection{Students' Perception of Radiographers at Clinical Settings}

In this study, respondents reported that during clinical training, they were satisfied with meeting their training objectives, enjoyed their time and worked as a team with very willing and available staff that assisted them in learning and this was revealed by $95.2 \%$ of the respondents. However, this research showed that not all training settings (supervisors and duty roaster) are available to provide students with a positive learning environment as reported by $38.1 \%$ of them [4]. This study has provided students with the necessary opportunity to reflect and examine issues or concerns on adequate and inadequate orientation to the work place, availability of assistance from staff members and so forth. Students only did not benefit from this exercise but the academics and industrial partners as well in the form of collaboration with agencies during the planning, allocation of resources and monitoring of clinical training as indicated by Dunn et al [5].

The challenge here is to maintain the quality of the training experience or improve on such experience. The response to the questionnaire showed that the majority of the student's impressions about the training were favorable, most importantly is that the students were of the view that they have benefited both theoretically and practically. While there may be some divergence in views held by faculty and 
industry partner, the most important consideration is to provide students the opportunity to experience their new knowledge on their perception and understanding of the work environment [6] Moreover, the focus or goal should be 'furthering the learning and development of (the) student, (and) not the needs of the placement organization' as cautioned by Hughes and Humphrey [7].

\subsection{Availability of Resources}

More than half of the respondents are of opinion that X-ray equipments and anatomical markers are inadequate at the department with indications as $52.4 \%$ and $42.9 \%$ respectively making students too clouded in a single duty room hence training students practically become very difficult as Adams, [8] stated that preparing students for entry into the health profession has always been a challenge to undertake. However, majority of the respondents $76.2 \%$ reported that cassettes and films were adequate. This revelation is very significant since cassettes are the main raw materials required during the training. Its availability makes clinical training effective and students are able to have adequate experience.

Again majority of the respondent (59.6\%) are of opinion that no monitoring devices are available for the students and they are too crowded in a room making radiation protection insufficient hence students not too willing to attend training in duty rooms. Clinical training dwells primarily on the availability of resources to make it more efficient and beneficial to the students. Inadequate resources means that fewer students will benefit from clinical training since the number of students in a particular examination room will far outnumber the equipment available. This will also mean that students will spend less time on the equipment and this can adversely affect the quality of clinical training they are receiving. Hughes [7] acknowledged that an encouraging and supportive clinical venue is conducive to positive learning outcomes, he proposed clinical training venue evaluation instrument to enable clinical trainings provide valuable learning experience of the 'real world', students undertaking them may have problems that need identifying and addressing.

Hughes' [7] reported the need for more 'empirical research into the characteristics of the workplace (examination room) as a learning environment'. Lave \& Wenger [9] argue that learning is a function of the 'activity, context and culture' in which it occurs. This is to say that learning is situated. By comparing with traditional classroom activities, knowledge is presented in context. Also, the social interaction involved in this setting is an important component of this type of learning. Learners become enmeshed in the training and acquire certain beliefs, training and behaviors promoted by the people in the training. The process is called 'legitimate peripheral participation', where beginners (i.e. radiography students) move from the periphery into the centre of the occupation (i.e. the radiography profession), and as students move towards the centre, they become more active and immersed within the radiography culture. With support and mentoring, the novices acquire the role and confidence to consolidate their practice.

\subsection{Imaging Modalities Allocated at the Clinical Settings}

Due to the inadequacy of specialized imaging modalities, majority of the respondents have been allocated to the general x-ray room (100\%) with only $33.3 \%$ and $31 \%$ having been allocated to the mammogram and dental imaging making training in this area very difficult and inadequate. In spite of the fact that most request are general in nature, radiographers are expected to have adequate knowledge and skills on all modalities. In a country like Ghana that the number of radiographers is not enough to cater for all the health centers, Radiographers find themselves performing certain specialized functions aside general x-rays. Most radiographers are alone in their districts and this requires that they are trained in all the areas of radiographic imaging.

Not having adequate clinical practice in these areas can have enormous effect on the delivery of quality health care to them. In a similar study, one of the deficiencies identified by students was the feeling of being inadequately prepared for clinical placement. This is an important aspect that was identified by some $22 \%$ of students. Student concerns about preparation, confidence and expectations about what they can do or cannot do need examining. Undoubtedly, there is a need to adequately prepare all students for placement. Hence, in addition to the current ways of preparing students for practice, some innovative approaches may need exploring. For instance, students might benefit from attending additional sessions, extra-coaching as heeded by Kaviani \& Stillwell, [10] the use of clinical challenge or contract, and use of more clinical laboratory simulation, which has been shown to improve transition of theory to practice [11].

Directing students to preceptors or clinical supervisors is another strategy suggested by many scholars [12, 13]. Preceptors and supervisors are valuable for professional and personal support and instruction [14]. These mechanisms have been shown to yield more productive and meaningful experiences and increase self-confidence among students.

\subsection{Time Allocation}

Majority of the respondents (74.4\%) did agree and strongly agree that 8day (4weeks) clinical duration was enough for each rotation in a particular duty room. This means each student must have visited each duty room four times in a semester during training. This suggested that though the four weeks clinical duration is enough, the hours should be more to help students acquaints themselves with the clinical environments as well as gain necessary skill.

Nonetheless, the amount of hours needed for clinical placement is still subject to debate as indicated by Penman \& Oliver [15]. If the resources are available and can effectively handle students coupled with the availability of supervisors then the time required for such a situation will be quite different from a situation where the numbers of students outnumber the resources and supervisors are not available. According to Penman \& Oliver [16] while students are now 
able to choose the specialty and location of their clinical placements, the amount of time and type of experience needed in the clinical setting is still subject to debate. He reported the trajectory of experience and the context of clinical learning in becoming competent in practice and has debunked the myth that more clinical hours are necessary to assist students in their clinical learning and gaining experience. Nonetheless, both tertiary education institutions and service areas are expected to collaborate in order to increase the learning opportunities for radiography students [17].

\subsection{Theory and Practice Gap During Clinical Training}

There were mixed views when respondents were giving their views on theory and practice gap. Some were of the view that there is no correlation between what is taught in class and what is practiced in clinical training rooms, while others think otherwise. The difference in theory and practice becomes evident during clinical practice examination where student is to handle a case alone unaided, because the student is used to the training rooms practice, he or she does that which is different from what he or she is taught theoretically, hence end up not doing well or even fails just because could not be able to justify or defend why that particular technique which is in disagreement with Sachdeva [18], that students should be able to give an account of why they selected a particular course of action and defend their position. Even the professional education causes recognize that it is difficult for students to make connection between theory, which is the focus of universities, and the practice, which takes place in clinical area.

\section{Conclusion}

The findings unraveled a number of challenges, some being lack of training equipment, plenty students in a small duty room without monitoring badges, practice and theory gap and the most obvious is the acquired information about how the students evaluate their clinical training rooms, was that majority of the students found their clinical training venues to be supportive of learning, professional growth, skill development and training. Their experiences with the clinical setting were pleasant and the outcome satisfying. The continuing emphasizing of the significant impact of context on student learning and satisfaction by the literatures on clinical training makes the outcome very interesting. The importance of feedback from all participants has been invaluable.

\section{Recommendations}

The study made provisions for the following recommendations.

This research suggests that various mechanisms and processes addressing the concerns of the minority of students who felt that they were ill-prepared for training and were dissatisfied with some clinical duty rooms will be put in place to solve such issues in the future. Again the research recommends using the same lecturers for both teaching theory and handling clinical areas and also, theory should be immediately followed by practical demonstration, improving facilities and time allocation are also recommended. More importantly, it should be agreed by all parties that theory and practice will continue to explore mechanisms and processes by which clinical training can be further improved and developed.

\section{References}

[1] Simpson M. (2000). Radiotherapy Service Review: Newcastle Mater Hospital. Newcastle

[2] Baume P. (2002). Radiation Oncology Inquiry. A vision for Radiotherapy. Canberra: Commonwealth of Australia.

[3] General Medical Council (2002). Tomorrow's Doctors. London, General Medical Council Ghana; Demographic and Health Survey (2008) In: Zunia knowledge Exchange UNCDF Smart Aid Research 2013

[4] Papp I., Markkanen M. \& Bonsdorff V.M. (2003). Clinical environment as a learning environment: student nurses' perceptions concerning clinical learning experiences. Nursing Education Today, 23: 262-268.

[5] DunnS V., Ehrich L., Mylonas A. \& Hansford B. (2000). Students' perceptions of field experience in professional development: a comparative study. Journal of Nursing Education, 39 (9): (393-400).

[6] Choi J. \& Hannafin M. (1995). Situated cognition and learning environments: roles, structures, and implications for design. Educational Technology Research and Development, 43(2), pp. 53-69.

[7] Hughes J. and Humphrey C. (1990). The policy context. In: Medical audit in general practice a guide to the literature. London: King's Fund Centre. In medical imaging and radiation oncology sciences, 1-4

[8] Adams V. (2002). Consistent clinical assignment for nursing students compared to multiple placements. Journal of Nursing Education, 41 (2): 80-82.

[9] Lave J., \& Wenger E. (1990). Situated Learning: Legitimate Periperal Participation. Cambridge, UK: Cambridge University Press.

[10] Kaviani N. \& Stillwell Y. (2000). An evaluative study of clinical preceptorship. Nursing Education Today; 20(3):218e26.

[11] Kember D. (2001). Reflective teaching and learning in the health professions. Oxford: Blackwell Science.

[12] King T. (2002). Development of student skills in reflective writing. UK: University of Portsmouth.

[13] Knights A.M. (1997). Radiography education and the profession. The Radiographer (September edition).

[14] Parsons TL, Dods J, \& Norman KE. (2006). Bridging the gap: MScPT student video-conferences enhance learning on clinical placement 
[15] Penman J. \& Oliver M. (2000). Meeting the challenges of assessing clinical placement venue in a bachelor of nursing programme. Nurse Educator, 8: 410-415.

[16] Penman J. \& Oliver M. (2004). Meeting the challenges of assessing clinical placement venues in a Bachelor of nursing programme. Journal of University and learning practice, 6: 59-73.
[17] Kulewicz S.J., (2001). Service learning: Head Start and a baccalaureate nursing curriculum working together. Pediatrics Nursing, 27(1): (37-43).

[18] Sachdeva A. K (1996). Use of effective feedback to facilitate adult learning. Journal of CancerEducation 11(2):106-118. 